\title{
Marcadores de Envolvimento Sistêmico no Lúpus Eritematoso Crônico Discóide $e^{(*)}$
}

\section{Markers of Systemic Involvement in Chronic Discoid Lupus Enythematosus}

\author{
Carolina Reato Marçon ${ }^{(1)}$, Mariane Regina de Moraes $^{(1)}$, Soraia Maria Lanzelotti ${ }^{(1)}$, \\ Débora Regina Cunha Simis ${ }^{(2)}$ e Gilberto Santos Novaes ${ }^{(3)}$
}

\section{RESUMO}

Objetivo: comparar as características clínicas e o perfil dos autoanticorpos entre o lúpus eritematoso crônico discóide (LECD) e o lúpus eritematoso sistêmico (LES), com o intuito de identificar algum marcador associado ao envolvimento sistêmico no LECD. Métodos: foram estudados 16 pacientes com LECD e comparados a 17 pacientes com LES, segundo os critérios do Colégio Americano de Reumatologia (ACR). Foram avaliadas as características demográficas, clínicas e pesquisados os seguintes autoanticorpos séricos, como fator antinúcleo (FAN), anti-DNA, anti-Sm, anti-Ro/SS-A, anti-La/SS-B, anti-ENA/RNP e anticardiolipina IgG e IgM. Nos pacientes com LECD foi realizada biópsia da pele e estudo histopatológico. Para comparação entre LES e LECD foi aplicado o teste do qui-quadrado. Foi considerado significativo $\mathrm{p} \leq 0,05$. Resultados: pacientes com LECD tiveram idade e duração da doença significantemente mais longa do que os pacientes com LES $(p<0,04)$. Não houve diferenças com relação ao número de critérios de diagnóstico de LES entre pacientes com LES e LECD. Entre os parâmetros clínicos, observouse associação da hipertensão arterial com a forma sistêmica de lúpus eritematoso $(p<0,03)$. Entre os autoanticorpos pesquisados somente o autoanticorpo anti-DNA mostrou estar significantemente associado ao diagnóstico de LES $(p<0,04)$. Conclusões: a análise dos dados clínicos e dos autoanticorpos no soro dos pacientes com LES e LECD identificaram hipertensão arterial e presença de anti-DNA no soro como os parâmetros que se mostraram associados ao envolvimento sistêmico no lúpus eritematoso.

Palavras-chave: lúpus eritematoso crônico discóide, lúpus eritematoso sistêmico, autoanticorpos, marcadores sistêmicos no LECD.

\begin{abstract}
Objective: to assess the clinical characteristics of CDLE, its interrelationship with SLE as well as the significance of some serum autoantibodies as markers of systemic involvement in CDLE. Methods: sixteen patients with CDLE were compared to seventeen patients with SLE according to the ACR criteria. Clinical and demographics characteristics and serum autoantibodies like antinuclear antibodies (ANA), antibodies to dsDNA, Sm, Ro/SS-A, La/SS-B, ENA/RNP, and anticardiolipin antibodies $\operatorname{Ig} G$ and $\operatorname{Ig} M$ were determined. The statistical evaluation was carried out using Chi-square test. A probability level of $\leq 0.05$ was considered significant. Results: age and disease duration in CDLE patients were significantly longer than in patients with SLE $(p<0.04)$. No difference was found regarding the number of ACR diagnostic and classification criteria between CDLE and SLE patients. Arterial hypertension was clearly associated with sistemic involvement $(p<0.03)$. Only antibody to dsDNA showed to be significantly associated with SLE diagnosis $(p<0.04)$. Conclusions: the clinical and laboratorial evaluation of CDLE and SLE patients showed that arterial hypertension and serum antibody to dsDNA were the laboratorial and clinical parameters associated to systemic involvement in CDLE.
\end{abstract}

Keywords: chronic discoid lupus erythematosus, systemic lupus erythematosus, autoantibodies, systemic markers in CDLE.

\footnotetext{
* Disciplina de Reumatologia da Pontifícia Universidade Católica de São Paulo (PUCSP), São Paulo-SP, Brasil. Recebido em 11/3/2004. Aprovado, após revisão, em $5 / 8 / 2004$

1. Bolsista do PIBIC-CEPE-PUCSP, São Paulo-SP, Brasil.

2. Professora doutora da disciplina de Dermatologia da PUCSP, São Paulo-SP, Brasil.

3. Professor titular da disciplina de Reumatologia da PUCSP, São Paulo-SP, Brasil.
}

Endereço para correspondência: Gilberto Santos Novaes. Rua Braz Laino 181, Jd. Emília, Sorocaba, SP, CEP 18031-030, Brasil. E-mail: gnovaes@terra.com.br 


\section{INTRODUÇÃO}

O lúpus eritematoso crônico discóide (LECD) é uma forma cutânea do lúpus eritematoso que acomete áreas expostas ao sol como face, couro cabeludo e orelhas. As lesões cutâneas se caracterizam por pápulas ou placas eritematosas, com descamação aderente e espessa, a qual oclui os folículos pilosos. Quando a descamação é removida sua base exibe pequenas excrescências que se correlacionam com a abertura dos folículos pilosos. Este aspecto se denomina de "tacha de carpete". A lesão duradoura desenvolve atrofia central, cicatriz e hipopigmentação, porém com freqüência apresenta borda eritematosa e às vezes elevada. Estas lesões persistem por anos e tendem a se expandir lentamente.

A histopatologia das lesões cutâneas do LECD mostra hiperqueratose, tamponamento folicular e atrofia da epiderme. A junção dermoepidérmica revela degeneração hidrópica dos queratinócitos basais e um infiltrado de células mononucleares que circundam os folículos pilosos e os vasos sangüíneos da derme. A microscopia de imunofluorescência direta mostra depósitos de imunoglobulinas e complemento na zona da membrana basal em aproximadamente $90 \%$ dos $\operatorname{casos}^{(1,2,3)}$.

Os pacientes com LECD em 5\% dos casos desenvolvem durante a sua evolução manifestações clínicas compatíveis com lúpus eritematoso sistêmico (LES) ${ }^{(2,4)}$. O diagnóstico do LECD é feito com base no quadro clínico e/ou histopatológico da lesão cutânea ${ }^{(5,6,7,8)}$. Em pacientes com LES lesões discóides são encontradas em até $8,82 \%$ dos $\operatorname{casos}^{(9)}$. O LECD pode existir como forma limitada, ou seja, lesões acima do pescoço, ou forma difusa com lesões acima e abaixo do pescoço ${ }^{(5)}$. Ainda que considerada pouco freqüente em LECD, Mayou et $a .^{(4)}$ detectaram a presença de autoanticorpos no soro de aproximadamente $60 \%$ dos pacientes com LECD.

Diversos autoanticorpos foram descritos no LES, como os anticorpos antinucleares (AAN), os quais são encontrados em mais de $95 \%$ dos pacientes com LES em atividade. Em mais de $60 \%$ dos casos, durante a evolução da doença, dois ou mais diferentes AANs podem ser identificados ${ }^{(1,2)}$. AANs podem ser encontrados em $40 \%$ a $60 \%$ dos pacientes com $\operatorname{LECD}^{(2,3,4)}$.

Anticorpos antifosfolípides e síndrome antifosfolípide (SAF) estão associados a variadas manifestações cutâneas, que podem ocorrer como primeira manifestação da síndrome. Entre as manifestações cutâneas associadas a SAF estão descritas livedo reticularis, vasculite necrosante cutânea, ulcerações cutâneas, púrpura, equimoses, nódulos dolorosos subcutâneos e hemorragias subungueais ${ }^{(1,10,11)}$. Estudos mostram ser variável a associação de LECD com anticorpos anticardiolipinas (ACL) ${ }^{(10)}$.
Na prática clínica há necessidade, em razão de implicações prognósticas e terapêuticas, da avaliação das manifestações sistêmicas e a determinação de autoanticorpos associados às lesões cutâneas discóides, principalmente nas formas discóides aparentemente isoladas. Desta forma justifica-se o estudo clínico e laboratorial do LECD nas suas correlações com o LES, com os marcadores sorológicos de doença lúpica e a presença de anticorpo anticardiolipina.

\section{PACIENTES E MÉTODOS}

Foram avaliados 16 pacientes com idade acima de 21 anos, com LECD e que estavam sendo acompanhados clinicamente nas disciplinas de Dermatologia e Reumatologia da Faculdade de Ciências Médicas de Sorocaba da PUC-SP. O estudo foi aprovado pelo Comitê de Ética em Pesquisa do Centro de Ciências Médicas e Biológicas da PUC-SP. Obteve-se o consentimento informado e esclarecido dos pacientes.

O diagnóstico de LECD foi realizado pelo aspecto clínico dermatológico das lesões cutâneas, complementado em todos os casos pela biópsia da pele, sendo o achado histopatológico compatível com LECD e com a exclusão de outras doenças cutâneas.

A avaliação clínica constou de anamnese, exame físico, doenças associadas (comorbidades) e exames laboratoriais com pesquisa de autoanticorpos no soro dos pacientes estudados. Os autoanticorpos pesquisados foram fator antinúcleo por imunofluorescência indireta (IFI) em células Hep-2, anti-DNA (IMFI em C. luciliae), anti-Ro/SS-A por ensaio imunoenzimático (ELISA), anti-La/SS-B (ELISA), anti-ENA/RNP (ELISA), anti-Sm (ELISA) e anticorpo anticardiolipina (ACL) IgG e IgM (ELISA). Os exames sorológicos foram realizados no Laboratório de Imunologia do Conjunto Hospitalar de Sorocaba (CHS). A biópsia da pele foi realizada na disciplina de Dermatologia por meio de técnica de "punch" e a coloração utilizada foi a hematoxilina-eosina $(\mathrm{HE})^{(12)}$.

O grupo de pacientes com LES foi constituído de 17 pacientes maiores de 21 anos de idade, com diagnóstico de LES conforme critérios de classificação e diagnóstico do Colégio Americano de Reumatologia (ACR) ${ }^{(13)}$, avaliados da mesma forma que o grupo com LECD, exceto quanto ao estudo histopatológico da pele, que não foi realizado nestes pacientes.

A análise dos resultados foi descritiva. Para comparação entre pacientes com LES e LECD foi aplicado aos resultados o teste de qui-quadrado. Foi considerado significativo $\mathrm{p} \leq 0,05^{(14)}$. 


\section{RESULTADOS}

A Tabela 1 mostra as características demográficas e clínicas dos pacientes com LES e LECD selecionados para este estudo. Nota-se que os pacientes com LECD têm idade e duração da doença significantemente mais longa do que os pacientes com LES $(p<0,04)$. Não houve diferença significativa com relação ao número de critérios do ACR de classificação e diagnóstico de LES, entre pacientes com LES e LECD, embora o grupo de pacientes com LECD apresente uma tendência a ter menor número de critérios presentes quando comparado com pacientes com diagnóstico definido de LES. Outras manifestações cutâneas e de mucosa que fazem parte dos critérios de diagnóstico de LES como rash malar, fotossensibilidade e úlcera oral não apresentaram diferença significante ou associação marcante com a forma discóide do lúpus. Fenômeno de Raynaud esteve presente apenas nos pacientes com a forma discóide da doença, mas não mostrou diferença estatística. A hipertensão arterial está claramente associada com forma sistêmica e agressiva de lúpus eritematoso $(\mathrm{p}<0,03)$ (Figura 1). A biópsia da pele realizada nos pacientes com LECD foi compatível com diagnóstico de LECD em 62,5\% dos pacientes. Nos demais pacientes o diagnóstico de LECD foi clínico e foram descartadas outras doenças no estudo histopatológico da pele.

A Tabela 2 mostra a freqüência de autoanticorpos no soro de pacientes com LECD comparados aos pacientes com LES. Entre os autoanticorpos pesquisados e comumente descritos como associados a formas sistêmicas do lúpus eritematoso somente o autoanticorpo anti-DNA, detectado pelo método de imunoflurescência indireta empregando como substrato a C. luciliae, mostrou estar significantemente associado ao diagnóstico de LES $(p<0,04)$ (Figura 2).

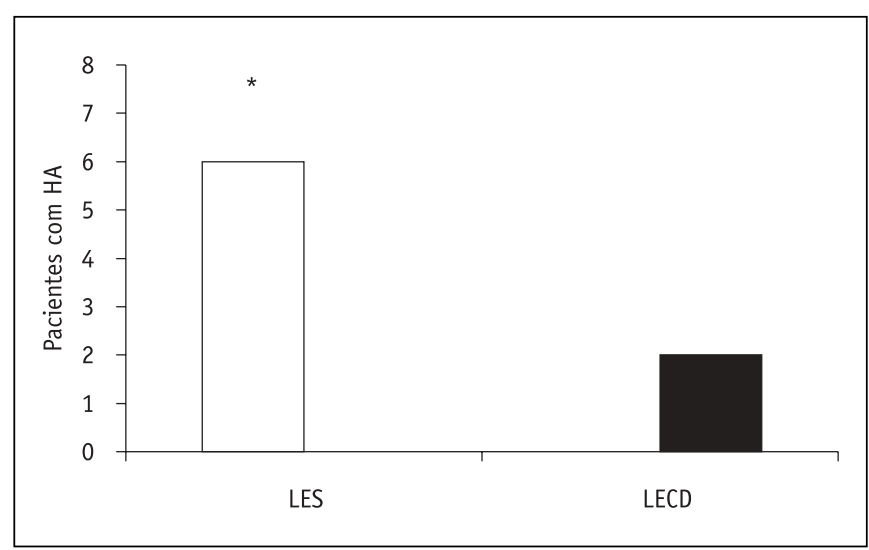

FIgURA 1 - Freqüência de pacientes com hipertensão arterial (HA) nos grupos de lúpus eritematoso sistêmico (LES) $=\square(n=6)$ e lúpus eritematoso crônico discóide (LECD) $=\mathbf{\square}(n=2) *{ }^{*} p<0,05$.
TABELA 1

CARACTERÍSTICAS DEMOGRÁFICAS E CLÍNICAS DE 17 PACIENTES COM LÚPUS ERITEMATOSO SISTÊMICO (LES) E 16 PACIENTES COM LÚPUS ERITEMATOSO CRÔNICO DISCÓIDE (LECD)

\begin{tabular}{lccc}
\hline & LES & LECD & $\mathbf{p}^{*}$ \\
\hline Idade (anos \pm DP) & $35,5 \pm 11,9$ & $43,3 \pm 9,7$ & 0.04 \\
\hline Sexo (\% feminino) & 100 & 93,7 & \\
\hline Duração da doença (anos) & $6,5 \pm 6,0$ & $10,7 \pm 5,7$ & 0.04 \\
\hline Critérios de LES (\%) & & & \\
$\quad 1$ a 3 critérios & 0 & 12,5 & \\
4 critérios & 41,1 & 31,2 & \\
5 a 7 critérios & 52,9 & 56,2 & \\
$>7$ critérios & 5,8 & 0 & \\
Rash malar (\%) & 70,5 & 75 & NS \\
\hline Lesão discóide (\%) & 0 & 100 & \\
Fotossensibilidade (\%) & 64,7 & 75 & NS \\
\hline Úlcera oral (\%) & 0 & 6,2 & NS \\
Artrite (\%) & 100 & 75 & NS \\
\hline Serosite (\%) & 35,2 & 18,7 & NS \\
Nefrite (\%) & 23,5 & 18,7 & NS \\
\hline Neuropsiquiátricas (\%) & 11,7 & 6,2 & NS \\
\hline Hematológicas (\%) & 70,5 & 37,5 & NS \\
\hline Hipertensão arterial (\%) & 35,2 & 28,5 & 0.03 \\
\hline Fenômeno de Raynaud (\%) & 0 & 57,1 & NS \\
\hline NS = não significante & & & \\
\hline
\end{tabular}

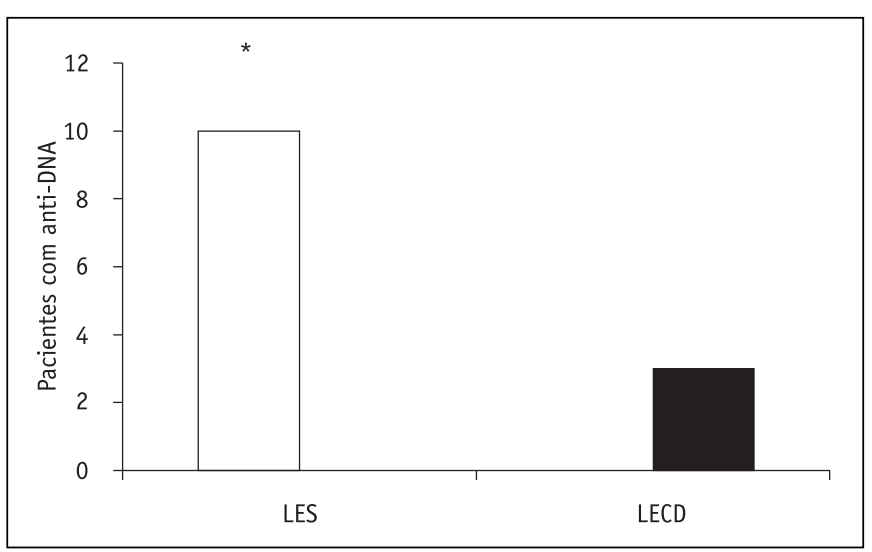

Figura 2 - Freqüência de pacientes com anticorpo anti-DNA positivo nos grupos de lúpus eritematoso sistêmico (LES) $=\square(n=10)$ e lúpus eritematoso crônico discóide $(\operatorname{LECD})=\mathbf{\square}(n=3) * p<0,05$. 
TABELA 2

FREQÜÊNCIA DE AUTOANTICORPOS NO SORO DE 17 PACIENTES COM LÚPUS ERITEMATOSO SISTÊMICO (LES) COMPARADOS A 16 PACIENTES COM LÚPUS ERITEMATOSO CRÔNICO DISCÓIDE (LECD)

\begin{tabular}{lccc}
\hline & LES & LECD & p* \\
\hline Fator antinúcleo (\%) & 58,2 & 43,7 & NS \\
\hline Anti-DNA (\%) & 58,8 & 18,7 & 0,04 \\
Anti-Sm (\%) & 23,5 & 12,5 & NS \\
Anti-Ro/SS-A (\%) & 35,2 & 12,5 & NS \\
Anti-La/SS-B (\%) & 17,6 & 18,7 & NS \\
Anti-ENA/RNP (\%) & 17,6 & 18,7 & NS \\
Anticardiolipina IgG (\%) & 41,1 & 25 & NS \\
Anticardiolipina IgM (\%) & 35,2 & 18,7 & NS \\
\hline
\end{tabular}

NS = não significante

\section{DISCUSSÃO}

Neste estudo, pacientes com LECD foram avaliados por meio de exame clínico e laboratorial com pesquisa de autoanticorpos no soro, e comparados com semelhante avaliação em pacientes com LES, visando determinar diferenças clínicas ou laboratoriais entre estas duas expressões clínicas da doença lúpica, as formas cutânea e sistêmica.

Nossos resultados mostram que os pacientes com LECD tiveram idade mais avançada e duração da doença mais prolongada do que os pacientes com a forma sistêmica da doença. A presença de lesões cutâneas compatíveis com LECD em um paciente com lúpus tem sido sugerida como marcador de uma doença lúpica de menor gravidade com manifestações renais ausentes ou discretas(6).

Apenas dois pacientes (12,5\%) com LECD não preencheram os critérios para diagnóstico e classificação de LES do ACR. Nos demais pacientes com LECD, em $87 \%$ dos casos, quatro ou mais critérios de LES estiveram presentes, em sua maioria $(56,2 \%)$ preenchendo cinco a sete dos 11 critérios para LES. No LES todos tinham quatro ou mais critérios, sendo que nove pacientes (52,9\%) tinham de cinco a sete critérios. Assim não observamos diferenças entre LECD e LES quanto aos critérios de diagnóstico e classificação de LES. Tem sido descrito que apenas $20 \%$ dos pacientes com LECD preenchem quatro ou mais critérios do ACR para o diagnóstico de $\mathrm{LES}^{(2)}$, o que nossos resultados não corroboram. O LECD pode preceder, ser concomitante ou seguir-se ao aparecimento de manifestações de LES $^{(6)}$.
Quanto aos diferentes acometimentos que compõem os critérios clínicos para classificação e diagnóstico de LES do $\mathrm{ACR}^{(13)}$ não encontramos diferenças significantes entre pacientes com LES e LECD. Envolvimento renal tem sido considerado como a variável de maior relevância estatística para a distinção entre pacientes com a forma cutânea (discóide e subaguda) da forma sistêmica ${ }^{(2)}$. Em nossos pacientes não confirmamos esta descrição. Alterações hematológicas foram mais freqüentes nos pacientes com LES $(70,5 \%)$ do que nos pacientes com LECD $(37,5 \%)$, porém não houve diferença estatística. Linfocitopenia tem sido descrita como marcador altamente sensível, porém de baixa especificidade, de envolvimento sistêmico no lúpus cutâneo $^{(15)}$. Fenômeno de Raynaud foi mais freqüente em pacientes com LECD $(57,1 \%)$ quando comparado aos pacientes com a forma sistêmica (0\%), porém também sem diferença estatística significante. Em razão de este estudo ter sido realizado em um único centro e não ser retrospectivo, o pequeno número de pacientes poderia explicar a não significância estatística do fenômeno de Raynaud.

Ao longo do tempo, pacientes com a forma discóide de lúpus podem desenvolver doença sistêmica ativa. Entre as manifestações clínicas, fotossensibilidade, alopecia, úlcera oral e fenômeno de Raynaud parecem sugerir pior prognóstico no lúpus cutâneo ${ }^{(16)}$. Outros estudos clínicos ${ }^{(6,17)}$ sugerem que a sistematização do lúpus eritematoso cutâneo esteja associada com a extensão das lesões cutâneas, com a presença de fenômeno de Raynaud, úlceras orais, fotossensibilidade e positividade de anti-DNA. Estudo comparando marcadores de atividade lúpica em pacientes com LES e lúpus cutâneo (discóide ou subagudo) verificou que nefrite, artrite e títulos altos $(\geq 1: 320)$ de FAN estavam entre os fatores de risco para envolvimento sistêmico no lúpus ${ }^{(2)}$.

Em nosso estudo, das variáveis clínicas apenas hipertensão arterial foi estatisticamente significante em pacientes com LES $(35,2 \%)$ quando comparados com LECD $(28,5 \%)$; $\mathrm{p}<0,03$. Hipertensão arterial tem sido considerada parâmetro clínico de gravidade e pior prognóstico em LES ${ }^{(18)}$. Neste estudo a presença de hipertensão arterial parece não estar relacionada de forma dependente com o dano renal, sendo possivelmente um fator independente de pior prognóstico para o LES.

A pesquisa de autoanticorpos no soro de nossos pacientes mostrou diferença significante $(\mathrm{p}<0,04)$ dos anticorpos antiDNA entre pacientes com LES $(58,8 \%)$ e LECD $(18,7 \%)$. Anticorpo anti-DNA tem sido considerado um autoanticorpo específico para o diagnóstico de LES. No entanto, sua sensibilidade é baixa, sendo positivo em apenas $50 \%$ a 
$60 \%$ dos pacientes com doença lúpica ativa, e sua presença está associada ao comprometimento renal. Parece ser raramente encontrado no LECD e tem sido demonstrado em 5\% dos casos de lúpus eritematoso cutâneo subagudo (LECSA $)^{(17)}$. Anticorpo anti-Sm também tem sido considerado específico de LES, porém é detectado em apenas $30 \%$ dos casos $^{(1)}$. Neste estudo encontramos anti-Sm em $23,5 \%$ dos pacientes com LES e em 12,5\% dos pacientes com LECD, freqüência próxima à descrita na literatura. Anticorpo anti-ENA/RNP está presente em aproximadamente $40 \%$ dos casos de LES, e foi demonstrado em apenas $17,6 \%$ de nossos pacientes com LES. Anticorpo antiRo/SS-A está presente em $40 \%$ dos pacientes com LES, e tem sido descrito também em pacientes com LECSA ${ }^{(19,20,21)}$. Anti-Ro/SS-A está presente em até 7,1\% dos casos de LECD $^{(19)}$, sendo um dos mais importantes autoanticorpos, junto com AAN, na avaliação de pacientes com hipótese diagnóstica de $\mathrm{LES}^{(1,21,22)}$. Anti-Ro/SS-A de 60 e $52 \mathrm{kDa}$ parecem ter pequena contribuição na patogênese da lesão

\section{REFERÊNCIAS}

1. Gladman DD, Urowitz MB: Clinical and laboratorial features of SLE. In: Klippel JH, ed. Primer on the rheumatic diseases. Atlanta: Arthritis Foundation, 251-7, 1997.

2. Tebbe B, Mansmann U, Wollina $U$, et al: Markers in cutaneous lupus erythematosus indicating systemic involvement. Acta Derm Venereol 77:305-8, 1997.

3. Gitlits VM, Sentry JW, Matthew MLSM, Smith AI, Toh ABH: Autoantibodies to evolucionarily conserved epitopes of enolase in a patient with discoid lupus erythematosus. Immunology 92:362-8, 1997.

4. Mayou SC, Wojnarowska F, Lovell CR, Asherson RA, Leigh IM: Anticardiolipin and antinuclear antibodies in discoid lupus erythematosus - their clinical significance. Clin Exp Dermatol 13: 389-92, 1988.

5. Callen JP: Chronic cutaneous lupus erythematosus. Clinical, laboratorial, therapeutic, and prognostic examination of 62 patients. Arch Dermatol 118:412-6, 1982.

6. Callen JP: Systemic lupus erythematosus in patients with chronic cutaneous (discoid) lupus erythematosus. Clinical and laboratory findings in seventeen patients. J Am Acad Dermatol 12:278-88, 1985.

7. David-Bajar KM, Bennion SD, DeSpain JD, Golitz LE, Lee LA: Clinical, histologic, and immunofluorescent distinctions between subacute cutaneous lupus erythematosus and discoid lupus erythematosus. J Invest Dermatol 99:251-7, 1992.

8. Chlebus E, Wolska H, Blaszczyk M, Jablonska S: Subacute cutaneous lupus erythematosus versus systemic lupus erythematosus: diagnostic criteria and therapeutic implications. J Am Acad Dermatol 38: 405-12, 1998.

9. Costallat LTL, Coimbra AMV: Lesões cutâneas no lúpus eritematoso sistêmico: estudo clínico em 272 casos. Rev Bras Reumatol 34: 14-8, 1994. discóide, ao contrário do demonstrado no lúpus neonatal ${ }^{(23)}$. Em nossa casuística, anti-Ro/SS-A ocorreu em 12,5\% dos pacientes com LECD e 35,2\% dos casos de LES.

A freqüência de anticorpos anticardiolipina em nossos pacientes com LECD não diferiu estatisticamente daquela observada em pacientes com LES. Anticorpos ACL têm sido detectados em até $40 \%$ dos pacientes com $\mathrm{LES}^{(1)}$. Anticorpos ACL tipo IgG têm sido demonstrados com uma freqüência variável de 39,4\% a 67,8\% nos pacientes com LECD, e ACL tipo IgM com uma freqüência de $15,2 \%$ a $50 \%$ nos pacientes $^{(24,25,26)}$. No entanto, pacientes com síndrome antifosfolípide raramente apresentam lúpus discóide sem evidência clínica ou sorológica de $\operatorname{LES}^{(10)}$. Há descrição entre nós de caso de lúpus discóide com fenômenos tromboembólicos associado a anticorpo antifosfolípide ${ }^{(11)}$.

No presente estudo a análise dos dados clínicos e dos autoanticorpos no soro de pacientes com LES e LECD mostram que hipertensão arterial e presença de anti-DNA foram os parâmetros associados ao envolvimento sistêmico no lúpus.

10. Gibson GE, Su WPD, Pittelkow MR: Antiphospholipid syndrome and the skin. J Am Acad Dermatol 36:970-82, 1997.

11. Innocencio RM, Kuhl VRF, Bonfiglioli R, Marques Neto JF, Provenza JR: Lúpus discóide com fenômenos tromboembólicos como evidência de sistematização. Rev Bras Reumatol 31:67-9, 1991.

12. Sampaio SAP, Rivitti EA: Dermatologia $2^{a}$ ed, São Paulo: Artes Médicas, 85, 2000.

13. Tan EM, Cohen AS, Fries JF, et al: The 1982 revised criteria for the classification of systemic lupus erythematosus. Arthritis Rheum 25:1271-7, 1982.

14. Swinscow TDV: Estatística um ao quadrado. São Paulo: Manole, 61-75, 1998.

15. Wenzel J, Bauer R, Uerlich M, Bieber T, Boehm I: The value of lymphocytopenia as a marker of systemic involvement in cutaneous lúpus erythematosus. Br J Dermatol 146:869-71, 2002.

16. Parodi A, Massone C, Cacciapuoti M, et al: Measuring the activity of the disease in patients with cutaneous lupus erythematosus. Br J Dermatol 142:399-400, 2000.

17. Parodi A, Caproni M, Cardinalli C, et al: Clinical, histological and immunopathological features of 58 patients with subacute cutaneous lupus erythematosus. A review by the italian group of immunodermatology. Dermatology 200:6-10, 2000.

18. Petri M, Spence D, Boné LR, et al: Coronary artery disease risk factors in the Johns Hopkins Lupus Cohort: Prevalence, recognition by patients, and preventive practices. Medicine 71:291-302, 1992.

19. Lee LA, Roberts CM, Frank MB, McCubbin BR, Reichlin M: The autoantibody response to Ro/SSA in cutaneous lupus erythematosus. Arch Dermatol 130:1262-8, 1994.

20. Magro CM, Crowson NA: The cutaneous pathology associated with seropositivity for anti-SSA (Ro): a clinicopathologic study of 23 adult patients with subacute cutaneous lupus erythematosus. Am J Dermatopathol 21:129-37, 1999. 
21. Provost TT, Watson R, Simmons-O'Brien E: Significance of the anti-Ro (SS-A) antibody in evaluation of cutaneous manifestations of a connective tissue disease. J Am Acad Dermatol 35:147-69, 1996.

22. Carazzai AM, Sucena RC, Milano SIM, Munhoz EGR, Santos RA, Scheinberg MA: Detecção do anticorpo SSA/Ro em pacientes com lúpus eritematoso sistêmico, lúpus eritematoso discóide e fotossensibilidade cutânea: estudo comparativo. Rev Paul Med 106:71-4, 1988.

23. Yoshimasu T, Hiroi A, Ohtani T, Uede K, Furukawa F: Comparison of anti 60 and $52 \mathrm{kDa} S S-A / R o$ antibodies in the pathogenesis of cutaneous lupus erythematosus. J Dermatol Sci 29:35-41, 2002.
24. Berth-Jones J, Hutchinson PE, Wicks AC, Warren J, Mitchell VE: Discoid lupus erythematosus associated with the anticardiolipin antibody syndrome (Letter). Br J Dermatol 120:469-70, 1989.

25. Ruffatti A, Veller-Fornasa C, Patrassi GM, et al: Anticardiolipin antibodies and antiphospholipid syndrome in discoid lupus erythematosus. Clin Rheumatol 14:402-4, 1995.

26. Tebbe B, Orfanos CE: Anticardiolipin antibodies in cutaneous lupus erythematosus and importance as a marker of vascular symptoms. Hautarzt 43:130-3, 1992 